December 2. In that assembly of more than six hundred, Jewish members of the Association, among whom are many who have been leaders of psychological thought, were conspicuous by their absenceand an opening address emphasised the demand for a psychology which expresses the genuine German spirit. Gestalt concepts were much in evidence and underlying all discussion was the assumption that parts are influenced by their membership character in larger wholes. It seems clear that "German psychology is developing a special concern for the type of Gestalt which is not limited to the perceptual field, but gives us the essential way of life of a whole personality". Of great practical significance was the address of Poppelreuter on political psychology. This directs attention to an increasing preoccupation with character and life as against intellect and theories and to the change of attitude brought about by the Hitler revolution, a change from an attitude of helplessness to one of determination to create, from disunity in economic ideas, political programmes, morality and world views to a sense of a common purpose. In the new whole of German culture, psychology no less than the other sciences must contribute to the realisation of the common purpose. "American psychologists," says Dr. Watson, "surveying the scene as spectators, may well wonder how long they can retain their own very considerable isolation from the major tasks of our generation." Coming as it does from the most influential focus of psychological doctrine in the United States, the comment is not without a certain piquancy.

\section{History of the Parsons Steam Turbine}

THE first issues for 1934 of the Engineer contain two instalments of a series of articles to be devoted to the development of the Parsons steam turbine. It is just fifty years since Sir Charles Parsons took out his patents for improvements in electric generators and improvements in rotary motors actuated by elastic fluid pressure, and constructed his first turbogenerator. This machine developed about $7 \cdot 5 \mathrm{kw}$. To-day single turbo-generators of $100,000 \mathrm{kw}$. capacity are in use, and the present estimated value

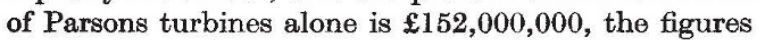
for marine and land turbines being $£ 92,000,000$ and $\mathfrak{£ 6 0 , 0 0 0 , 0 0 0}$ respectively. Nothing to equal that rapid extension of value and dimensions has ever happened before. The publication of the series of articles will take many weeks and will appropriately mark the jubilee of this great invention. The first article contains a detailed description of the original turbo-generator, while the second, after referring to the early development of the parallel-flow turbines, deals with the radial-flow turbines of 1889-91.

\section{Automatic Voltage Control of Electrical Systems}

THe problems which arise in connexion with maintaining the voltage of supply constant in electrical distributing systems have been closely studied by electrical engineers during recent years. The per- missible variation allowed by the regulations is plus or minus four per cent of the 'declared' voltage, but the average regulation is much closer; the houses near the supply station at times of maximum load are supplied at a voltage above the declared pressure and the few near the ends of the distributing mains at a voltage below the normal. The lamps near the supply station therefore give a better light and have a shorter life than the distant lamps. In a paper read by W. Kidd and J. L. Carr to the Institution of Electrical Engineers on December 7, methods of automatic voltage regulation and switch control were described. The city of Manchester is the first area to have complete automatic voltage regulation and it also has supervisory control for its main sub. stations. By a careful application of automatically controlled regulators it is shown that the voltage on distribution networks can easily be maintained within the permissible limits. Manual control of voltage is never quite satisfactory and necessitates the uneconomic employment of additional labour. The installation of the regulating equipment gives better service to the public and removes a possible cause of complaint. It has to be remembered that a rise or fall in the voltage of one per cent increases or diminishes the light emitted by the lamp by about three per cent. Several diagrams are given showing methods of adapting existing transformers by means of automatically controlled tap-changing devices so that the voltage of supply can be kept constant at all loads.

\section{Organisation of a Social Centre}

IN a recently issued publication entitled "The Centre" (London : P. S. King and Son, Ltd. 3d.) various problems confronting the organisers of social centres for the unemployed are discussed by five contributors, who have had experience in the running of such centres. Special emphasis is laid on the importance of not losing sight of the individual in the mass, and of adapting the facilities provided to the needs of the individual. To prevent employables from becoming unemployable, though most desirable, is but a small part of a centre's activity. The way social centres can help best is to aid the individual to discover new powers, since this is the key to re-creation and progress. One person may need opportunities for thinking (study, reading, talks or discussions) ; another may wish to make something ; another may prefer some form of amusement (a game, dancing, gymnastics) ; others again may want advice as to cooking food, mending boots, making clothes or keeping well. It is no part of the purpose of a centre to compete with or undercut existing employment. It is conceivable that certain articles such as handwoven scarves might find a ready market, but the repeated manufacture of products of the same kind is not the aim of a social centre. The work there should be undertaken for the sake of the effect which the making has upon the maker. Its purpose is to perfect the individuals and this will not be obtained by encouraging them to do the same thing over and over again. 